\title{
Fall Monitoring Device for Old People based on Tri- Axial Accelerometer
}

\author{
Jing Luo ${ }^{1}$ \\ School of Electronic and Electrical \\ Engineering \\ Shanghai University of Engineering \\ Science Songjiang District \\ Shanghai 201620, China
}

\author{
Bocheng Zhong ${ }^{2}$ \\ School of Electronic and Electrical \\ Engineering \\ Shanghai University of Engineering \\ Science Songjiang District \\ Shanghai 201620, China
}

\author{
Dinghao $\mathrm{Lv}^{3}$ \\ School of Electronic and Electrical \\ Engineering \\ Shanghai University of Engineering \\ Science Songjiang District \\ Shanghai 201620, China
}

\begin{abstract}
To be able to timely and effective judgment of the elderly fall, a fall monitoring device based on tri-axis accelerometer for elderly is designed. The device collects acceleration and the angle between elderly and horizontal plane of elderly people by MPU6050 tri-axial accelerometer, comparing the acceleration and angle that people and horizontal plane with threshold value to determine whether the old people fell. Delay for a period of time compared the angle and threshold again to judge the elderly in the fall still, finally send text messages to a mobile phone of guardian by GPRS module so that elderly can be helped.
\end{abstract}

Keywords-Fall monitoring; tri-axis acceleration sensor; threshold; GPRS module

\section{INTRODUCE}

Fairly rapid development of modern society an aging population is more and more serious. There are many reasons caused the problem such as public health level advances in technology have made extended life expectancy, production and living rhythm is fast, the birth rates is falling caused by life pressure, and so on. The outlook of world population report released by the United Nations learned that the current global showed a trend of aging population. Aging also is not a disease of the wealthy in the developed countries, developing countries also began to appear aging, and one of the most important reasons is low fertility rate overall global. These countries like United States, Japan, Korea, Australia and other western countries have unprecedented pension burden of an aging population. More and more elderly need to live in the compound and there are a lot of family support function weakened. To this problem, this paper designed a fall monitoring device that can be carried easily. This device can collect acceleration signals and angle signals and then through these signals to judge whether the elderly is fall, then the GPRS that controlled by the Microcontroller can send a message to guardian phone. The device can help the old man in the empty nest for rescue when they are in the fall.

Detection of falls using accelerometers and mobile phone technology [1-2] proposed a device that has two components: an intelligent mobile phone and an accelerometer. The accelerometer is responsible for collecting the acceleration signal and smartphone is responsible for judge whether the elderly is fall by acceleration threshold and send a message to guardian, but In daily life has many more vigorous exercise acceleration will influence the judgment of the threshold algorithm such as rapid squat, so we cannot judge whether the elderly is fall by a single acceleration threshold. Accelerometer Placement for Posture Recognition and Fall Detection [3] put accelerometers in the four parts of body to detect human body falls and distinguish the gesture. However, too many accelerometers in the body can lead to less mobile when the elderly in action. Implementation of a real-time human movement classifier using a triaxial accelerometer for ambulatory monitoring [4] is to perform the vast majority of signal processing onboard the wearable unit using embedded intelligence, the system distinguishes between periods of activity and rest, recognizes the postural orientation of the wearer, detects events such as walking and falls, and provides an estimation of metabolic energy expenditure.

Real-Time Fall Detecting System Using a Tri-axial Accelerometer for Home Care [5] proposes a real-time detection system based on home, this system can distinguish between up to four different stumble like forward, backward, turn right and left, and it easy to carry, low cost and high accuracy. But only through accelerometer can't accurate judgment too violent action as quick squat, quick sit, et al. In order to ensure the security of the old people under the condition of not to affect the normal life of elderly, many researchers are working on better fall monitoring devices. But there are lots of problems in the elderly fall monitoring device, we should consider improving all aspects of the problem in safety, quickly, conservation and charges et al. This system adopted a tri-axial acceleration MPU6050 to collect the acceleration signal and angle signal of elderly action, then used the improved algorithm to judge the elderly fall, finally send the message of fall to guardian by GPRS module, so that rescue the elderly in time.

\section{FALL DETECTION SYSTEM}

\section{A. System Architecture}

The elderly fall monitoring system based on tri-axial acceleration sensor uses tri-axis acceleration sensor gather the acceleration and angle signal of the activity of old people and transmission to Microcontroller using threshold algorithm to judge whether the elderly people fall, last using a serial port sent AT command [6] control fall in the GPRS module to send message of old people falling to the guardian for help. System architecture is shown in figure 1: 


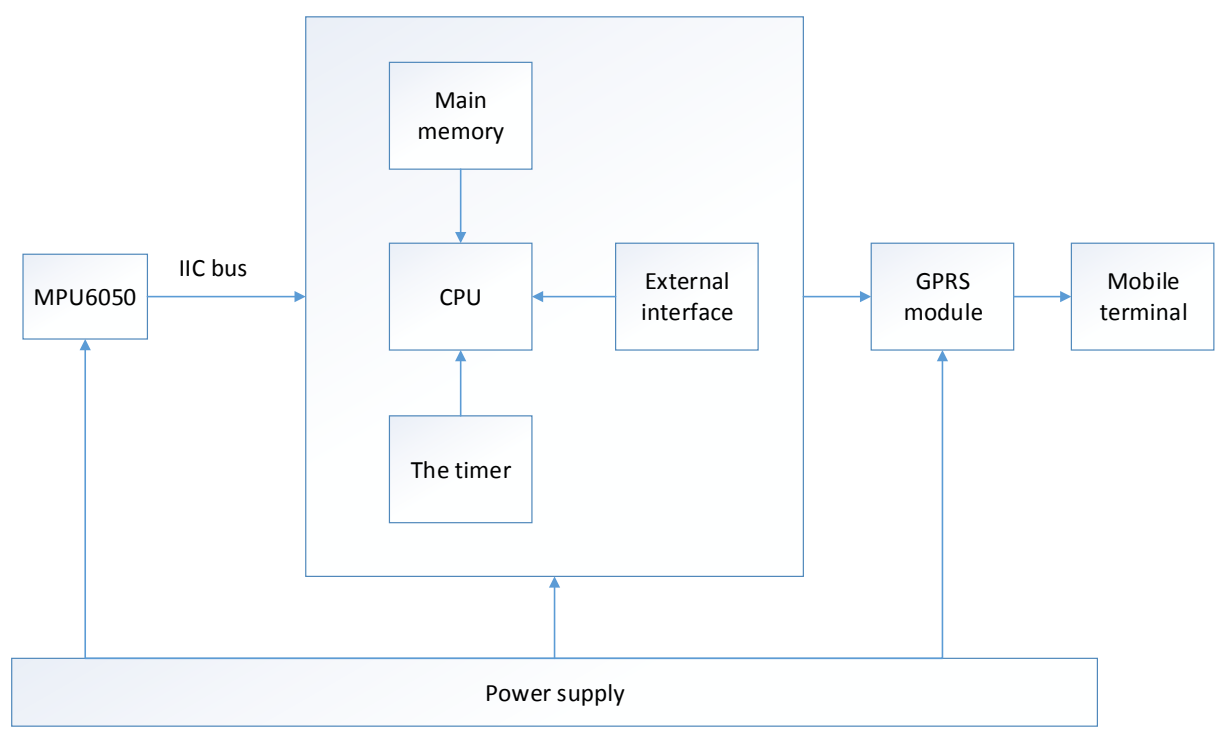

Fig. 1. System architecture

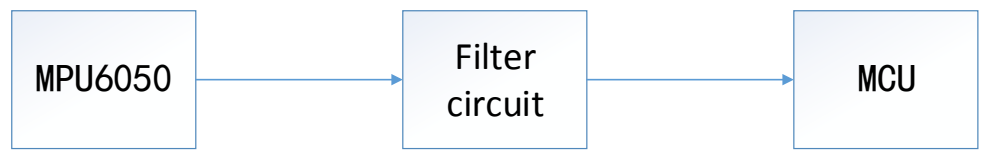

Fig. 2. signal acquisition and processing module

\section{B. The Signal Acquisition And Processing Module}

Signal acquisition and processing module of fall monitoring device is composed of three parts, respectively, tri-axis acceleration sensor MPU6050, filter circuit and microcontroller. Signal acquisition and processing module structure diagram as shown in figure 2.

The acceleration signal acquisition module using the triaxis acceleration sensor MPU6050 collecting activities acceleration of the elderly. Tri-axis acceleration sensor MPU6050 the integration of the three axis accelerometer, gyroscope and extensible digital motion processor [7]. It can collect the acceleration and angular velocity signal in direction of $\mathrm{X}, \mathrm{Y}$ and $\mathrm{Z}$ at the same time. It avoids the acceleration scheduler and the problem of the difference between the timeline of gyroscope, reduces the effect of the preset [8], sensor drift and reduce the complicated data fusion algorithms, motion processing operation of the operating system loads.

The MCU of data processing module is stm32f103rbt6, it be used to receive the acceleration and angle signals that be collected by tri-axis acceleration sensor and carry on the judgment, in the final analysis to see if the elderly fall and in the danger. Stm32f103rbt6 [9]adopt high-performance ARM Cortex - M3TM32 RISC core that can operate at $72 \mathrm{MHZ}$ frequency, have high speed embedded memory and support three kinds of low power consumption mode. It can be achieve a best balance between low power consumption with short start time.

\section{The Wireless Communication Module}

The fall monitoring device of elderly people selects and uses the GPRS wireless communication module to send the message of old people falling, for the sake of this information of elderly falling can be transmitted to guardian accurately and promptly. The GPRS wireless communication module that a mode of packet-switched data load and transmission is developed on the system of GSM [10]. By using the TCP/IP protocol, The GPRS wireless communication module can ensure the data's security and reliability in the transmission process of data. The way of GPRS wireless communication is channel multiplexing that can always keeping online and to avoid the serious consequences that brought by dropping. GPRS communication module is being paid by amount of information, that can be cut down the costs of users who using it.

\section{SOFTWARE DESIGN OF FALl MONITORING DEVICE}

This system with the aid of VC platform to write programs related that can control the MPU6050 to obtain the activities' acceleration and angle of elderly and estimate the outcome that dealing with the improved threshold algorithm. The fall monitoring device acquisition acceleration and angular velocity signal through the tri-axis acceleration sensor. The angle that between the elderly and horizontal plane can be get by integral the angular velocity. The angle that by transformation to obtain and acceleration are transmitted to $\mathrm{MCU}$ to processing and judgement by threshold algorithm. Once the result of judgement is the elderly fall, the MCU controlling the GPRS wireless communication module to send a text message to the guardian to rescue the elderly immediately.

\section{THE FALl MONITORING AlgORITHM}

The acceleration can be considered as a three dimensional vector that acceleration signal of the elderly activities is gathered by tri-axis acceleration sensor. It will be said on the space rectangular coordinate system, and $\mathrm{X}, \mathrm{Y}, \mathrm{Z}$ axis 
represents the direction of the acceleration. Owning to the body center of gravity direction is unpredictable, and the single direction of acceleration cannot judge whether the body falls, so threshold algorithm is based on the resultant acceleration (SVM) [11] as the basis of judgment. The SVM is expressed as

$$
\operatorname{SVM}=\sqrt[2]{\left(a_{x}^{2}+a_{y}^{2}+a_{z}^{2}\right)}
$$

The SVM is acceleration amplitude only associated with the intensity of exercise, has nothing to do with direction. Only according to the acceleration signal cannot accurately judge whether the elderly fall, in order to decrease the misjudgment.

In this paper, combining the acceleration and the angle between human body with horizontal coordinates with up to judge the elderly fall status, Moment of the experiment shows the body fell to the ground impact acceleration reach maximum
SVM, at the moment the angle of human body and horizontal coordinates is greater than 60o. This threshold algorithm will delay $2 \mathrm{~s}$ after judgment fall again to judgment of Angle, to eliminate the circumstance that after elderly people fell can autonomous action and have no influence for normal activities. Algorithm process as shown in figure 3.

Combined the acceleration threshold judgment with angle threshold of the human body and horizontal coordinates can reduce misjudgment caused quickly squat action and so on. It can cut down the number of alarm for the elderly fall without any hurt and reduce the work burden of guardians that determine the size of angle and threshold again after a 2 s delay. By tri-axial acceleration sensor acquisition the status SVM signal of human body such as: static, walking, running, fast squats, bend over and fall as shown in figure 4 .

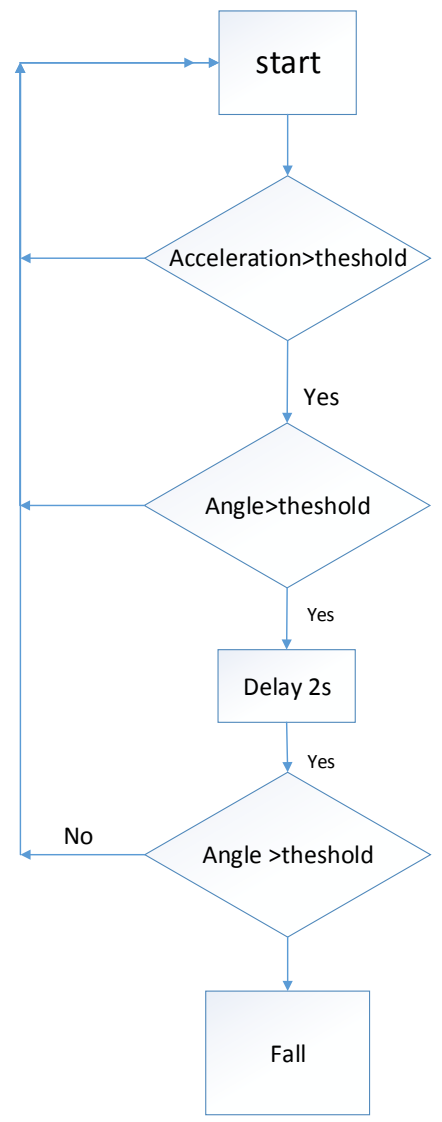

Fig. 3. Algorithm process 


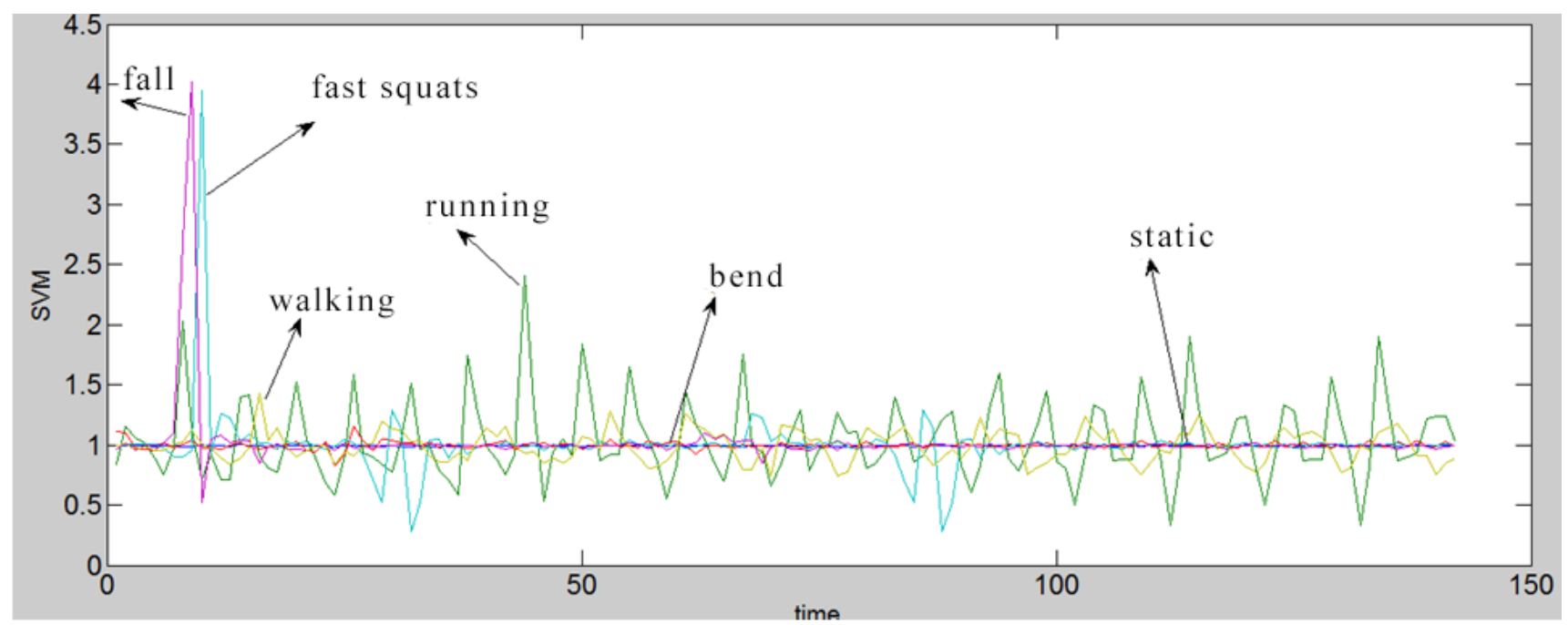

Fig. 4. 4 SVM

From the figure we can see the SVM of human in static is range from $0.98 \mathrm{~g}$ to $1 \mathrm{~g}$, Resultant velocity only affected by the acceleration of gravity, it is consistent with the actual activity. The SVM of walk and bend down have a minor changes are range from $0.5 \mathrm{~g}$ to $1.5 \mathrm{~g}$. Due to when elderly people is running have a certain degree of ups and downs that the action is more dramatic than walking and bend down, so its SVM to a maximum of $2.4 \mathrm{~g}$. And fast crouch down and fell the most severe serious in daily actions weightlessness, and the SVM can up to $4 \mathrm{~g}$. $3 \mathrm{~g}$ can be selected as the SVM threshold to judge fall preliminary by decide in the experiment. From the figure 4 shows there are many actions like quickly squat and other will cause misjudgment in acceleration threshold value judgment, so only by judge the SVM cannot be completely accurate to judge whether the elderly fall, this paper puts forward a method that combine to the acceleration threshold algorithm and the angle threshold algorithm of the old people and the horizontal value of $\mathrm{X}, \mathrm{Y}$ axis to judge whether the elderly fall. The angle that the human body and $\mathrm{X}, \mathrm{Y}$ axis in horizontal direction of static, running, fast squat, fall, bend shown in figure 5-6.

Figure 5-6 shows the body at stationary state the angle that the human body and the horizontal $\mathrm{X}, \mathrm{Y}$ direction is not more than 10 , it is a small fluctuations belong to deviation that human caused when he stationary, and it does not affect the judgment falls. In the process of running and fast squat the angle is rang from 150 to $25 \mathrm{o}$ and always in fluctuation, it indicating that the human body has a range of shaking in the activity, this is consistent with the normal behavior of the human body. Experiment enumerates the angle that can be get by simulation the human fall forward and fall to right, setting the angle between the body and $\mathrm{X}$ axis toward the side down, with the $\mathrm{Y}$ axis angle of toward the forward and backward. The figure shows that when the elderly fall its angle between human body and horizontal plane is more than $60 \mathrm{o}$, according this angle to judge if the elderly is fall and he fall forward or toward the side. The algorithm which angle threshold combined with acceleration threshold can estimate whether the elderly fall accurately, ruled out the misjudgment caused by some actions such as rapid squat and other movement acceleration greater than the threshold. The algorithm will judge the size of angle and threshold that human under the current state and $\mathrm{X}, \mathrm{Y}$ axis to confirm old people fall and lose the ability to act autonomously after a microcontroller judgement determine the elderly is fall.

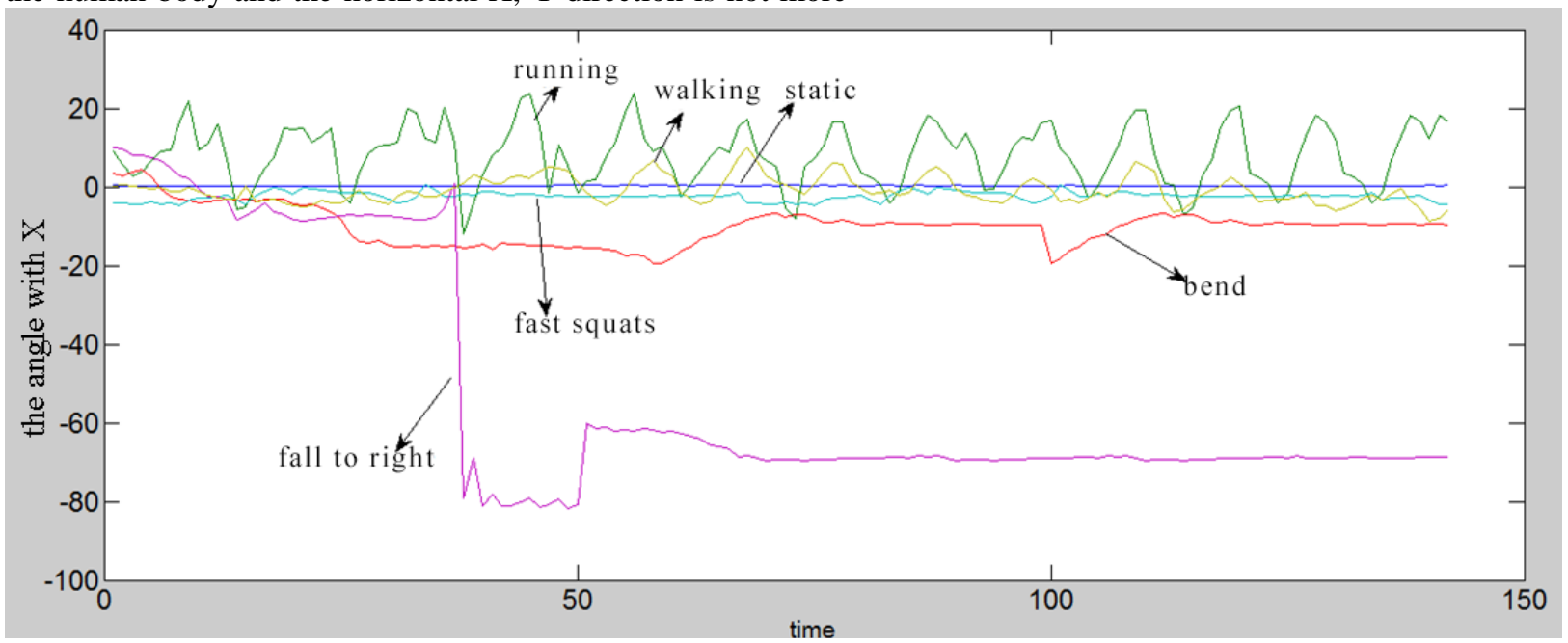

Fig. 5. The angle with $X$ 


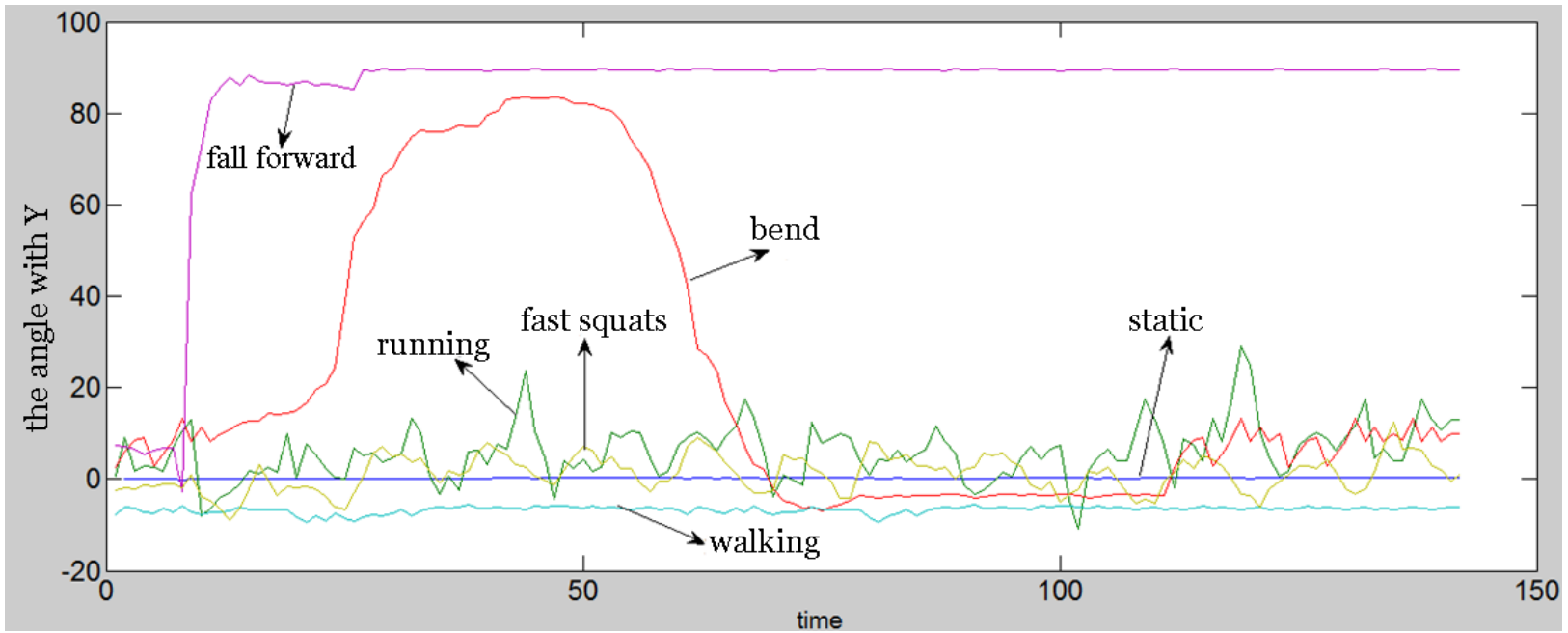

Fig. 6. The angle with $\mathrm{Y}$

\section{EXPERIMENTAL RESULTS AND ANALYSIS}

Taking into account the physical condition of the elderly, the experiment used a ground of 20 to 25 years old young people to the imitate activities of old people. Experimental determines whether the old people fall and occurs some injuries though the acceleration and angle have been collected that in the actions like walking, running, fast squat, bend down and fall.
In the process of experiment, the young people simulated many activities of elderly, we can see there have some false positives if judged only by acceleration threshold, it cannot identify fast squat and other actions that the SVM greater than threshold whether is fall. The acceleration threshold comparison results are shown in figure 7.

The angle threshold comparison results are shown in figure 8 and 9.

\begin{tabular}{cccccccc}
\hline Action & Static & Walk & Run & Fast squat & Bend & Fall forward & Fall to side \\
\hline SVM>threshold & - & - & - & yes & - & yes & yes \\
\hline Send message & - & - & - & yes & - & yes & no \\
\hline False positives & - & - & - & yes & - & no \\
\hline
\end{tabular}

Fig. 7. The acceleration threshold comparison results

\begin{tabular}{|c|c|c|c|c|c|c|c|}
\hline Action & Static & Walk & Run & Fast squat & Bend & Fall forward & Fall to side \\
\hline angle $>$ threshold & - & - & - & - & - & - & yes \\
\hline Send message & - & - & - & - & - & - & yes \\
\hline False positivies & no & no & no & no & no & no & no \\
\hline
\end{tabular}

Fig. 8. The angle with $\mathrm{X}$ axis comparison results

\begin{tabular}{|c|c|c|c|c|c|c|c|}
\hline Action & Static & Walk & Run & Fast squat & Bend & Fall forward & Fall to side \\
\hline angle $>$ threshold & - & - & - & - & yes & yes & - \\
\hline Send message & - & - & - & - & - & yes & - \\
\hline False positivies & no & no & no & no & yes & no & no \\
\hline
\end{tabular}

Fig. 9. The angle with $\mathrm{Y}$ axis comparison results 


\begin{tabular}{|c|c|c|c|c|c|c|c|}
\hline Action & Static & Walk & Run & Fast squat & Bend & Fall forward & Fall to side \\
\hline angle>threshold & - & - & - & yes & - & yes & yes \\
\hline Angle with $X>$ threshold & - & - & - & - & - & - & yes \\
\hline Angle with $\mathrm{Y}>$ threshold & & & & & yes & yes & - \\
\hline Send message & - & - & - & - & - & yes & - \\
\hline False positivies & no & no & no & no & no & no & no \\
\hline
\end{tabular}

Fig. 10. Combine the acceleration and angle comparison results

By the experiment result, acceleration threshold and angle threshold comparison all can produce false positives. This paper combined the acceleration and angle threshold algorithm can distinguish the elderly fall accurately in the fast squat and other actions that acceleration greater than acceleration threshold, and the rate of false positives to 0 . The device can comparative the size of angle and threshold again delay $2 \mathrm{~s}$ after judgement in the state of fall by combined algorithm to analysis whether the old people fall and lose the ability to act independently, according to the specific situation of the elderly micro controller will control the GPRS module send text messages to a guardian mobile phones. After many experiments proved that the device can accurately diagnose fall and normal send text messages to mobile phones.

\section{CONCLUSION}

This paper puts forward a fall monitoring devices, using MPU6050 tri-axial acceleration sensor to collect the acceleration and angle of elderly, through the threshold detection algorithm to judge whether the elderly fall and cause certain harm, in a timely manner through GPRS module send text messages to a guardian mobile phone timely treatment to help.

The device has some advantage: it has a small volume and convenient to carry; it has a low cost and can judge the situation of fall timely and effectively. The innovation of this device is use the MPU6050 tri-axial acceleration sensor, the monitoring method within can judge whether the elderly fall down and in danger, finally microcontroller controls the GPRS module send text messages to a guardian mobile phone. The device can judge in the timely and reduced the workload of the guardian and guardian has no necessary to worry about it.

\section{REFERENCES}

[1] Lee, Raymond, Carlisle, Alison. Detection of falls using accelerometers and mobile phone technology[J]. Age and Ageing, 2011, 40 (6): 690-696.

[2] Jiangpeng Dai, Xiaole Bai, Zhimin Yang; et al. PerFallD: A pervasive fall detection system using mobile phones[C]//8th IEEE International Conference on Pervasive Computing and Communications Workshops, 2010: 292-297.

[3] Gjoreski, H, Lustrek, M, Gams, M. Accelerometer Placement for Posture Recognition and Fall Detection[C]//Intelligent Environments (IE), 2011 7th International Conference on. IEEE, 2011: 47-54.

[4] Karantonis, D.M.; Narayanan, M.R; Mathie, M; et al. Implementation of a real-time human movement classifier using a tri-axial accelerometer for ambulatory monitoring $[\mathrm{J}]$. Information Technology in Biomedicine, IEEE Transactions on, 2006, 10(1): 156-167.

[5] Chien-Cheng Lan, Ya-Hsin Hsueh, Rong-Yuan Hu. Real-time fall detecting system using a tri-axial accelerometer for home care[C]//Biomedical Engineering and Biotechnology (iCBEB), 2012 International Conference on. IEEE, 2012: 1077-1080.

[6] Zhan-bei Wang. Design of Soil Temperature and Humidity Remote Monitoring System of Tea Garden Based on GPRS [J]. Journal of Agricultural Mechanization Research, 2013, 356(6):973-976.

[7] ZhijianYin, Haojie Ning, Inoue Y, et al. A novel wireless motion sensor for analyzing golf swing[C]//SENSORS, 2013 IEEE. IEEE, 2013: 1-4.

[8] Popelka V. A Self Stabilizing platform[C]//Control Conference (ICCC), 2014 15th International Carpathian. IEEE, 2014: 458-462.

[9] Ting-zhen Dai, jun-hui Hu. A control system for the ultrasonic 3-DOF micro/nano manipulator[C]//Piezoelectricity, Acoustic Waves, and Device Applications (SPAWDA), 2014 Symposium on. IEEE, 2014: 25 28.

[10] Rashdi Adnan, Malik Rafia, Rashid Sanam, et al. Remote energy monitoring, profiling and control through GSM network[J]. Arabian Journal for Science and Engineering, 2013, 38(11): 3249-3257.

[11] Kwolek, Bogdan, Kepski, Michal. Human fall detection on embedded platform using depth maps and wireless accelerometer $[\mathrm{J}]$. Computer Methods And Programs In Biomedicine, 2014, 117(3):489-501. 